\title{
Construction of a Talent Cultivation Mode for Traditional Chinese Medicine Nursing Inheritance
}

\author{
Ye $\mathrm{LI}^{1, \&}$, Jing $\mathrm{LIU}^{2, \&}$, Ling TANG ${ }^{3}$, Chun-Mei ZHANG ${ }^{2}$, Mi ZHANG ${ }^{2}$ \\ ${ }^{1}$ Otolaryngology, Head and Neck Surgery, Peking University First Hospital, Beijing, 100034, China; ${ }^{2}$ Outpatient \\ Department, Beijing University of Chinese Medicine Third Affiliated Hospital, Beijing, 10029, China; ${ }^{3}$ Department of \\ Nursing, Dongfang Hospital of Beijing University of Chinese Medicine, Beijing 100078, China
}

\begin{abstract}
Objective: To explore and build traditional Chinese medicine (TCM) nursing inheritance on the talent training scheme, the foundation for cultivating outstanding talents of TCM nursing inheritance.

Methods: The modified Delphi method was used for conducting two rounds of consultation with 15 TCM nursing experts to formulate training plan.

Results: The questionnaire recovery rates of two rounds of expert enquiry were $100.00 \%$, with high experts enthusiasm, experts coefficients were 0.83 and 0.81 , respectively. Kendall coordination coefficient were 0.26 and 0.30 , respectively $(P<0.01)$. After two rounds of expert consultation, the TCM nursing inheritance talent training scheme was finally formed, including 3 first-level indicators, 7 second-level indicators and 24 third-level indicators.

Conclusion: Construction of talent cultivating program of TCM nursing inheritance is scientific and feasible, with a certain reference value.
\end{abstract}

Key words: Traditional Chinese medicine nursing; Inheritance; Talent cultivation

\section{Introduction}

TCM nursing is an important component of TCM and an important part of the nursing field in China, which integrates TCM culture and modern natural science culture. In ancient China, there is no full-time nursing staff, and Chinese medicine nursing methods and experience fails to form a inheritance theoretical system. With the evolution of medicine pattern and the unique advantages of TCM nursing on the techniques and

\section{\&Those authors contributed to the work equally.}

Author profile :

Ye LI, otolaryngology head and neck surgery, Peking University First Hospital, Beijing, 100034, China Email:89173838@qq.com;

jing LIU, outpatient department, Beijing University of Chinese Medicine Third Affiliated Hospital, Beijing, 10029, China

Received: July 25 2019, Revised: September 16 2019, Accepted: October 102019 ,

Online: November 152019 methods, the development of TCM nursing is more and more attention. Meanwhile, The national institutes of health clearly put forward to carry forward the traditional technology of TCM. During the development of TCM nursing, the talents cultivation of TCM nursing inheritance is crucial. How to cultivate new TCM nursing talents with solid basic theory and knowledge of TCM nursing, strong inheritance ability of TCM nursing, TCM nursing thinking ability, practice ability and innovation ability to improve the overall level of TCM nursing, is a subject that needs to be continuously explored in TCM nursing stuides ${ }^{[4]}$. Based on the inheritance model of famous old Chinese medicine doctors (the inheritance from master to apprentice), this study established the relationship between teachers and 
apprentices, followed the TCM nursing experts into TCM nursing clinic, summarized and studied the academic thoughts of TCM nursing experts, and formed the talent training plan of TCM nursing inheritance according to the characteristics of TCM nursing.

\section{Materials and Methods}

\section{General information}

The convenience sampling method was adopted to select 15 experts of TCM clinical nursing management, nursing education and nursing scientific research from Beijing University of Traditional Chinese Medicine and some 3A-level TCM hospitals for expert consultation.

Inclusion criteria for experts: clinical TCM nursing experts require bachelor's degree or above, work in 3A-level or above hospitals, associate chief nurse or above, and clinical teaching for more than 5 years; Nursing education experts are required to have a master's degree or above master's tutor, with more than 5 years of experience in TCM nursing education; they have high authority and representativeness, and can reflect the high credibility of the research results.

\section{Methods}

Establishment of research group: The research team consists of 10 members, including 1 TCM doctor, 2 master of nursing, 6 clinical TCM nursing experts, and 1 graduate student of master of nursing. Team members are responsible for formulating expert consultation questionnaire, selecting consultation experts, and conducting statistical analysis of the returned data.

Preparing questionnaire for expert letter consultation: On the basis of literature research and discussion of the research group, the questionnaire of expert consultation was preliminarily formed. 5 experts engaged in clinical nursing and nursing education of traditional Chinese medicine were selected for pre-experiment. Through expert consultation and subject group discussion, the content and structure of the initial questionnaire were adjusted to form a formal questionnaire. The questionnaire consists of two parts. The first part is the questionnaire of experts, including experts' age, length of service, education background and professional title. The second part is the evaluation table of the indicator system of TCM nursing inheritance talent cultivation program, including 3 dimensions of medical skill inheritance, medical book inheritance and medical spirit inheritance. Opinions and suggestions from experts on the establishment and importance of sub-items of each dimension are solicited.

Expert letter consultation: In this study, two rounds of expert letter consultation were conducted, which were conducted through E-mail and paper questionnaires distributed on site. The results of expert letter consultation and feedback were sorted out and analyzed, and the contents of the questionnaire were adjusted to make the results more scientific and consistent. Prior to the consultation, contact the expert to explain the purpose and significance of this research, and conduct the consultation after obtaining his/her consent.

\section{Statistical analysis}

EXCEL was used to double input data and check, SPSS 20.0 statistical software was used for statistical analysis, and the frequency and percentage of statistical description were used for the analysis of experts' general data and self-evaluation results. The positive coefficient of experts was evaluated according to the recovery rate of the questionnaire. The authority degree of experts was expressed by the weight of academic level, the basis of experts' judgment and the familiarity of the investigated questions. The coordination degree of expert opinions was expressed by the coefficient of variation.

\section{Results}

\section{Basic information of experts}

The 15 experts were all from Beijing, all female, and the ages of the experts were 35-54 years old, with an average age of $(42.35 \pm 3.18)$. They had 8-30 years working experience in related field, average $(20.13 \pm 4.26)$ years. There were $2(13.33 \%)$ doctor, $4(26.67 \%)$ master, $9(60.00 \%)$ bachelor; $8(53.53 \%)$ Senior title, and $7(46.67 \%)$ intermediate title . 


\section{Positive coefficient of experts}

Two rounds of expert letter consultation were conducted in this study, and the recovery rate was $100.00 \%$ and the effective rate was $100.00 \%$. The effective recovery rate of the questionnaire is $>70 \%{ }^{[5]}$, indicating that experts are highly motivated to participate. In the first round of consultation, 5 experts proposed the modification, and in the second round of consultation, 1 expert proposed the modification.

\section{Coefficient of expert authority}

Expert authority index $\mathrm{Cr}=(\mathrm{Ca}+\mathrm{Cs}) / 2$. The authority coefficient of the first round of letter consultation was $0.83(\mathrm{Cs}=0.84, \mathrm{Ca}=0.82)$. The authority coefficient of the second round of correspondence consultation was 0.81 $(\mathrm{Cs}=0.75, \mathrm{Ca}=0.87)$. The authority coefficients of the two rounds of letter consultation experts were $>0.70^{[6]}$, indicating that the authority of the experts was high and the result of the correspondence consultation was credible.

\section{Degree of expert coordination}

Kendall coordination coefficient was used to evaluate the degree of expert coordination, which ranges from 0 to 1 . The coordination coefficient reflects the degree of coordination of experts on the evaluation of indicators, and the larger the value is, the better the coordination degree is ${ }^{[7]}$. The Kendall coordination coefficient of the first round of letter consultation in this study was 0.26 , and the Kendall coordination coefficient of the second round of letter consultation was 0.30 , both of which were statistically significant $(P<0.01)$. The coordination coefficient of the second round was higher than that of the first round, indicating that the coordination of experts gradually improved in the process of correspondence consultation.

\section{Result of letter inquiry}

After two rounds of expert consultation, the talents training program for TCM nursing inheritance was finally determined, including 3 first-level indicators, 7 secondlevel indicators and 24 third-level indicators, covering medical skill inheritance, medical book inheritance and medical spirit inheritance index system. See Table 1.

\section{Discussion}

\section{Consultation analysis of the training program}

This study conducted two rounds of expert consultation, and formed an indicator system of TCM nursing inheritance talent cultivation program including 3 firstlevel indicators, 9 second-level indicators and 24 thirdlevel indicators, including medical skill inheritance, medical book inheritance and medical spirit inheritance. The positive coefficient of experts refers to the degree to which experts care about and cooperate with research, and is the key issue of expert consultation. The higher the positive coefficient is, the more valuable the consultation results are. The positive coefficient of experts in this study was expressed by the questionnaire recovery rate, and the recovery rate of the 2 rounds of correspondence consultation was $100.00 \%$, indicating that the positive coefficient of experts was very high, proving that the research results of this study had a high value. In addition, the authority coefficient of the two rounds of expert correspondence consultation in this study is 0.83 and 0.81 respectively, and it is generally believed that the authority coefficient of the expert $>$ is acceptable at $70 \%$, which indicates that this study has good authority.

\section{Paying attention to TCM nursing talent training and training mode}

The inheritance, development and innovation of TCM nursing is a long-term systematic project related to the fate of TCM nursing, which is the primary task facing the development of TCM nursing at present ${ }^{[8]}$. To make TCM nursing can fully exert its features and advantages, promote the TCM nursing personnel specialization, standardization, lifelong overall cultivation, strive for TCM nursing talents into focus on the medical basic theory, operation skills of TCM, scientific research ability of the TCM college nurse ${ }^{[9]}$, improve the ability of TCM nursing, Beijing Municipal Administration of Traditional Chinese Medicine officially launched on May 12, 2015, Beijing TCM nursing inheritance studio station construction, set up min TCM nursing inheritance studio, TCM nursing GuiMeiFen inheritance studio, Feng Yunhua TCM nursing inheritance studio and workstations. The training system of this study takes the nursing experts 
Table 1 Indicator system of TCM nursing inheritance talent training program

\begin{tabular}{|c|c|c|}
\hline Significance & Score $(\bar{x} \pm s)$ & Variation coefficient \\
\hline 1. Medical skill inheritance & $4.96 \pm 0.27$ & 0.02 \\
\hline 1.1 Learning with teachers & $4.88 \pm 0.33$ & 0.07 \\
\hline 1.1.1 Operation skills of TCM nursing & $4.80 \pm 0.41$ & 0.09 \\
\hline 1.1.2 TCM nursing professional knowledge & $4.88 \pm 0.33$ & 0.07 \\
\hline 1.1.3 Thinking mode of dialectical nursing & $4.84 \pm 0.37$ & 0.08 \\
\hline 1.1.4 Communication skills & $4.90 \pm 0.44$ & 0.08 \\
\hline 1.2 Nursing rounds & $4.82 \pm 0.28$ & 0.06 \\
\hline 1.2.1 Teaching ability & $4.68 \pm 0.59$ & 0.12 \\
\hline 1.2.2 Organizational capacity & $4.48 \pm 0.56$ & 0.13 \\
\hline 1.2.3 Coordination ability & $4.68 \pm 0.59$ & 0.12 \\
\hline 1.3 Lectures by famous scholars & $4.80 \pm 0.50$ & 0.10 \\
\hline 1.4 Assessment index & $4.96 \pm 0.43$ & 0.08 \\
\hline 1.4.1 Basic knowledge & $4.88 \pm 0.33$ & 0.07 \\
\hline 1.4.2 Operation skill & $4.88 \pm 0.33$ & 0.07 \\
\hline 1.4.3 Room inspection notes & $4.50 \pm 0.65$ & 0.13 \\
\hline 1.4.4 Record with teacher & $4.56 \pm 0.42$ & 0.13 \\
\hline 1.4.5 Case analysis & $4.74 \pm 0.46$ & 0.10 \\
\hline 2. Medical book inheritance & $4.94 \pm 0.36$ & 0.04 \\
\hline 2.1 TCM classics & $4.74 \pm 0.49$ & 0.15 \\
\hline 2.1.1 Inner longitude of the yellow emperor & $4.56 \pm 0.51$ & 0.11 \\
\hline 2.1.2 Compendium of materia medica & $4.56 \pm 0.51$ & 0.11 \\
\hline 2.1.3 Typhoid miscellaneous disease theory & $4.56 \pm 0.51$ & 0.11 \\
\hline 2.2 Dialectical nursing & $4.68 \pm 0.48$ & 0.10 \\
\hline 2.3 Assessment index & $4.96 \pm 0.43$ & 0.08 \\
\hline 2.3.1 Reading notes & $4.50 \pm 0.65$ & 0.13 \\
\hline 3. Medical spirit inheritance & $4.88 \pm 0.43$ & 0.05 \\
\hline 3.1 Medical ethics education & $4.72 \pm 0.46$ & 0.09 \\
\hline 3.1.1 Great medical practitioners are sincerely & $4.80 \pm 0.41$ & 0.08 \\
\hline 3.1.2 Major medical practice & $4.80 \pm 0.41$ & 0.08 \\
\hline 3.1.3 Introduction to Chinese traditional culture & $4.44 \pm 0.62$ & 0.10 \\
\hline 3.1.4 History of ancient Chinese thoughts & $4.42 \pm 0.71$ & 0.16 \\
\hline 3.2 Assessment index & $4.96 \pm 0.43$ & 0.08 \\
\hline 3.2.1 Ideological quality & $4.66 \pm 0.76$ & 0.16 \\
\hline 3.2.2 Humanistic quality & $4.68 \pm 0.59$ & 0.12 \\
\hline 3.2.3 Vocational quality & $4.66 \pm 0.76$ & 0.16 \\
\hline 3.2.4 Professional quality & $4.72 \pm 0.46$ & 0.09 \\
\hline
\end{tabular}

of famous old Chinese medicine as the cornerstone, breaks the traditional learning mode, implements the "mentorship" training mode (the inheritance from master to apprentice) of TCM nursing talents, and carries out the lecture of "experience inheritance of nursing experts of famous old Chinese medicine", laying a certain foundation for the inheritance of TCM nursing.

\section{Improving the curriculum training system, highlighting the characteristics of TCM nursing}

At present, the training mode of TCM nursing talents 
basically refers to or imitates the western medicine nursing education system, without highlighting the characteristics of TCM nursing in curriculum setting, textbook construction, teaching methods and continuing education $^{[10]}$. This study focus on the reform of the system, on the basis of TCM nursing professional teaching mode, "mentorship type" teaching; on the content, focus on TCM classics, nursing based on syndrome differentiation, and the teaching of Chinese traditional culture such as course; in terms of skills, focuses on the cultivation and training of TCM nursing thinking ability and clinical practice ability; in terms of ideas, pay attention to the education of humanistic quality and medical ethics. At the same time, a series of lectures on the clinical experience of famous TCM nursing experts are held, requiring students to complete at least $5 \mathrm{TCM}$ classic reading notes, so as to make the training objectives have distinct practicality.

\section{Enriching teaching means and paying attention to teaching effect}

Sun Simiao, a medical expert in the Tang dynasty, once said, "one who is good at thinking (differentiating syndromes) and rational use of drugs is a good doctor", which emphasizes the importance of clinical thinking ${ }^{[11]}$. TCM Clinical thinking refers to the thinking activities in judging and analyzing the nature of disease, the law of onset, formulating the principles of treatment and prevention of disease, and the process of syndrome differentiation and nursing ${ }^{[12]}$. Ward round following the tutors is the organic combination of TCM theories and practical experience, which focuses on training TCM clinical thinking of specialty nurses. When students were training, the tutors analyze the collected data by inspecting, listening and smelling, asking and taking pulse of patient in bed to determines the diseases. Through such real cases, they explain the relevant TCM basic theories and analyse TCM etiology and pathogenesis to students, and finally guide nursing based on differentiating syndrome ${ }^{[13]}$. This method is adopted in the form of interaction to enhance the students' active participation. In the process of training, teachers show the students the main way of TCM clinical thinking, ask with each other, increase students' enthusiasm to participate, inspire the students' emotional experience and learning enthusiasm, gradually cultivate the dialectical thinking mode of the nursing.

\section{Combination of mentorship education and clinical practice}

The trainees will stay at the TCM nursing inheritance studio for at least 6 months, and the tutor will fully guide the trainees in the period of their study and clinical practice, so as to cultivate the students' practical ability of TCM nursing as early as possible. The trainees must complete at least 50 notes during the study with the teacher, make their rounds with the teacher at least once a week, and write the record of ward rounds, and combined with the tutor's academic experience, they can put forward new opinions and ideas. At the end of the study, the trainees should complete one paper about their tutor's academic thoughts and technical expertise or TCM nursing research.

\section{Application prospect of TCM nursing inheritance talent training program}

The development of nursing career is linked to the level of nursing education and specialized level of its synchronous development ${ }^{[14]}$. TCM nursing, as traditional medicine in China, has a long inheriting history. However, being shocked by the western medicine nursing education, its services and advantages is gradually degraded and weakened. Lack of TCM nursing talents is the important cause for hindering inheritance and development of TCM nursing characteristics ${ }^{[15]}$. Therefore, it is very necessary to cultivate TCM nursing inheritance talents and promote the development of TCM nursing career. The talent training of TCM nursing inheritance has just started, and the social research on the talent training mode of TCM nursing inheritance is insufficient, lacking of perfect training system, so the training effect is not satisfactory. Therefore, it is necessary to carry out positive publicity to help nursing staff to recognize the advantages and characteristics of TCM nursing, establish a standardized and scientific training system, form a benign incentive mechanism, give full play to the potential of TCM nursing inheritance staff, and promote the long-term development of TCM nursing. 


\section{Conclusion}

This study constructed the index system of TCM nursing inheritance on the talent training scheme, after two rounds of expert enquiry, TCM nursing inheritance talent training scheme is eventually formed, containing three first-level indicators, 9 secondary indexes, 24 three indicators, expert opinions are consistent, authority coefficient is higher, the results are scientific and reliable, which provides theoretical reference for the construction of a united TCM nursing inheritance talent cultivation program in the future. In addition, This study is only a preliminary construction of the talents training system of TCM nursing inheritance, and there are limitations in the breadth and depth of the study. In future studies, the training system needs to be further discussed.

\section{Declaration}

The authors of this article declare no conflict of interest.

\section{References}

1 Shen Q. Influencing factors and countermeasures of clincial application of TCM nursing techniques. Chin J Nurs, 2010, 45(3): 265-267.

2 Jin $\mathrm{XC}$, Luo HO, Tan QF, et al. Practice and experience of Shicheng system education. Chin Med Mod Distance Educ China, 2013, 11(2): 66-67.

3 Yang X, Wang YL, Bai XL, et al. Opportunities and challenges for the development of traditional Chinese medicine nursing under the new health reform. J Nurs Admin, 2016, 16(8): 542-544.

4 Tao Y, Jiang AL. Preliminary study of cultural ecology basis of traditional Chinese medicine nursing inheritance. J Nurs Training, 2011, 26(20): 18401842.

5 Jiang XP, Yan L, Zheng XL, et al. Study on cultivation objectives and curriculum system of nursing postgraduate constructed by Delphi method. Chin Nurs Res, 2015, 29(11): 1307-1311.

6 Lin J, Fang S, Xiong L, et al. Discussion on core competence indicator system master degree candidate in traditional Chinese medicine nursing professional degree. Hunan J Trad Chin Med, 2016, 32(11): 123126.

7 Li Y. Study on applicability selection of rehabilitation technology for home movement dysfunction of stroke patients in convalescence stage. Beijing: Beijing University of Chinese Medicine, 2014.

8 Tao Y. A new mode of teaching. Shanghai: Second Military Medical University, 2013.

9 Wang JY, Tang L, Li Y. Existing problems and prospects of traditional Chinese medicine nursing talent cultivation. Chin J Mod Nurs, 2013, 19(27): 3432-3434

10 Li Y, Tang L. Study on influencing factors of development of TCM nursing. Chin Nurs Res, 27(17): 1675-1676.

11 Zhu X, Feng YX. Old doctor of traditional Chinese medicine Zhang Jiemei understanding and use of the thought "To be a doctor, to be aware of Yi". Chin J Chin Med, 2015, 30(2): 210-212.

12 Wang YY, Zhang HM, Wang YP. Clinical thinking model and behavioral paradigm of traditional Chinese medicine. J Beijing Univ Trad Chin Med (Clinical Medicine), 2012, 19(2): 1-2.

13 Mo XW, Wang B, Zhou CY. The Chinese medicine clinical teaching model: veteran doctors of TCMcentric model. Educ Chin Med, 2018, 37(3): 55-57.

14 Bai YM, Song YL, Wang QQ, et al. Study for the training program of specialty nurses in traditional Chinese medicine. J Nurs Training, 2014, 29(23): 2136-2139.

15 Xie WH, Bai YM, Zhou X. The progress and thinking of traditional Chinese medicine nursing. Shanxi J Trad Chin Med, 2011, 27(3): 51-53. 